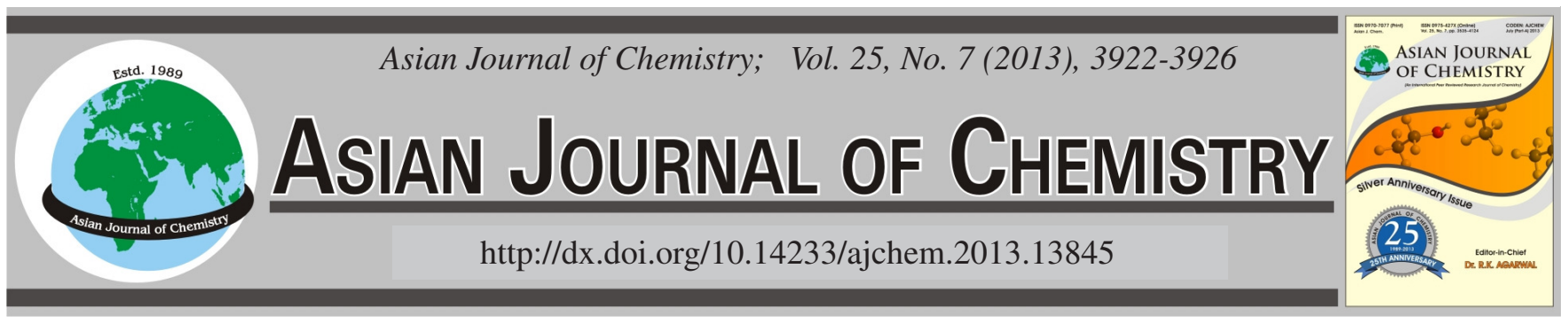

\title{
Preparation and Evaluation of a Molecularly Imprinted Sol-Gel Hybrid Material for Selective Solid-Phase Extraction of Sarafloxacin in Milk
}

\begin{abstract}
Dong Zhang ${ }^{1,2}$, Yun-Kai Lv ${ }^{1, *}$, Rong CHeN $^{2}$ and Chen-Chen ShI ${ }^{1}$
${ }^{1}$ College of Chemistry and Environmental Science, Hebei University, Baoding 071002, P.R. China

${ }^{2}$ The Central Institute for Correctional Police, Baoding 071002, P.R. China

${ }^{3}$ Yingtan institute of Measurement and Testing Technology, Yingtan 335000, P.R. China

*Corresponding author: Fax:+86 312 5079628; Tel: +86 312 5079359; E-mail: lvyunkai@hbu.edu.cn

A new molecularly imprinted organic-inorganic hybrid material was prepared by sol-gel process in aqueous phase system based on the interaction of ion pair and hydrophobic between the sarafloxacin and the functional monomer. Sarafloxacin was used as the template molecular, 3-aminopropyltriethoxysilane as functional monomer and methyltrimethoxysilane as cross-linking agent. Synthesis and adsorption conditions were optimized for obtaining sorbent with the high adsorption capacity, selectivity, hardness and toughness. Binding study demonstrated that the imprinted hybrid composites showed excellent affinity and high selectivity to sarafloxacin. The present imprinted sorbent was successfully applied for the solid-phase extraction of fluoroquinolone antibiotics in milk. The mean recoveries of sarafloxacin and lomefloxacin in milk were 75.6-94.2 \% and 61.73-88.56\%, respectively, with relative standard deviations (RSDs) of 3.56-5.21\%. The limits of detection ( $\mathrm{LOD}, \mathrm{S} / \mathrm{N}=3$ ) and the limits of quantitation (LOQ, $\mathrm{S} / \mathrm{N}=10$ ) of the proposed method were 0.010 and $0.0333 \mathrm{mg} \mathrm{kg}^{-1}$ for sarafloxacin hydrochloride, 0.012 and $0.04 \mathrm{mg} \mathrm{kg}^{-1}$ for lomefloxacin hydrochloride, respectively.
\end{abstract}

Key Words: Organic-inorganic hybrid material, Molecularly imprinted polymer, Solid-phase extraction, Sarafloxacin, Milk.

\section{INTRODUCTION}

Antibiotics are widely employed in animal husbandry. They are used both for the prophylaxis and the treatment of diseases and as feed additives to promote mass gain. Antibiotics can be added directly to food products (mainly to milk) to prolong their freshness ${ }^{1}$. All these cases raise the occurrence of the veterinary drug residues in animal tissue and milk intended for human consumption. US Food and Drug Administration (FDA), European Union (EU) and Chinese Ministry of Agriculture have established a maximum residue limit (MRL) of $0.1 \mathrm{mg} \mathrm{kg}^{-1}$ for fluoroquinolone antibiotics in milk ${ }^{2-4}$. These limits require the development of sensitive and selective methods for antibiotic residues in food to guarantee the safety of food.

Some methods have focused on the determination of fluoroquinolone multiresidues in milk $^{5-7}$ and sample pretreatment such as liquid-liquid extraction ${ }^{6,8}$, solid-phase extraction $(\mathrm{SPE})^{7,9-11}$ and matrix solid-phase dispersion technique ${ }^{12}$. Solidphase extraction is being widely utilized as a preconcentration and separation technique because of high enrichment factors, flexibility, absence of emulsion, easy of automation and safety with respect to hazardous samples. As the adsorbents play an important role in SPE, much of the current research in SPE focus on the development of new adsorbents ${ }^{13}$. Among them, molecular imprinting technique is one of the most attractive and efficient synthetic method.

Molecularly imprinted adsorbents exhibit high affinity and selectivity but they may shrink or swell when exposed to different organic solvent and thus considerably cause the deformation of the molecularly imprinted polymers (MIPs) receptors and decrease the recognition ability towards the template. An alternative approach is the preparation method of molecularly imprinted sol-gel material (MISGM) that can offer excellent mechanical strength and good solvent resistance in the presence of the inorganic matrix. Molecularly imprinted sol-gel material is fabricated by a conventional sol-gel process and incorporation of the template molecules into rigid inorganic or inorganicorganic networks. After removal of the template, molecular cavities with distinct pore size, shape, or chemical functionality remain in the cross-linked host ${ }^{14}$.

Herein, we report a simple and straightforward strategy for the preparation of molecularly imprinted hybrid material (MIP-HM) through a conventional sol-gel process and application of SPE of fluoroquinolone antibiotics from the milk samples. The optimal synthesis conditions and solid-phase extraction conditions were investigated. The obtained MIP-HM 
was evaluated and applied as selective adsorbent to SPE-HPLC for fast enrichment and efficient separation of the sarafloxacin and the analogues from the milk samples.

\section{EXPERIMENTAL}

Lomefloxacin hydrochloride (LOM) and sarafloxacin hydrochloride (SAR) were purchased from National Institutes for Food and Drug Control (Fig. 1). 3-Aminopropyltriethoxysilane (APTES) and methyltrimethoxysilane (MTMS) were purchased from Nanjing Lianye Chemical Co., Ltd. (Shanghai, China). All the other chemicals were of the analytical or the HPLC grade and used without further disposal. Doubly deionized water (DDW) was used throughout. Samples for HPLC were filtered through a $0.45 \mathrm{~mm}$ membrane filter.
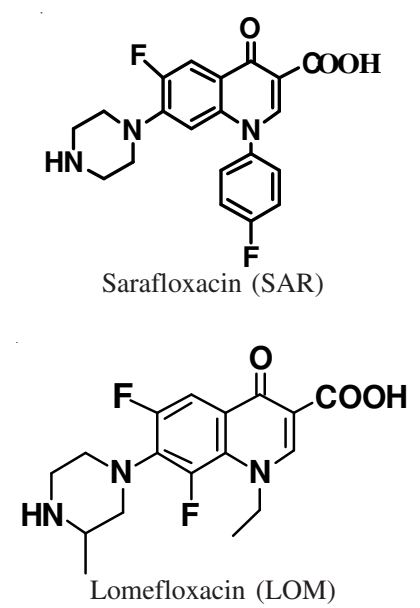

Fig. 1 Structures of fluoroquinolones

HPLC analysis was performed in using a liquid chromatography system containing a LC-20AT pump, a SPD-20A UVVIS detector (Shimadzu, Japan). The analytes were separated in a Venusil $\mathrm{XBP} \mathrm{C}_{18}$ column $(250 \mathrm{~mm} \times 4.6 \mathrm{~mm}, 5 \mu \mathrm{m})$ from Bonna-Agela Technologies (Tianjin, China). The mobile phase was methanol-acetonitrile- $0.1 \%$ trifluoroacetic acid solution (MeOH-ACN-0.1\% TFA, 8:20:72, v/v) and the flow rate was $1.0 \mathrm{~mL} \mathrm{~min}{ }^{-1}$ at $25^{\circ} \mathrm{C}$. Aliquots of $10 \mu \mathrm{L}$ were injected into the column and the chromatograms were recorded at $280 \mathrm{~nm}$.

Preparation of the SAR-imprinted sorbent: Sarafloxacin (77.6 mg, $0.2 \mathrm{mmol}$ ) was dissolved in $10 \mathrm{~mL}$ of ethanol in flask and mixed with APTES ( $1 \mathrm{~mL}, 4 \mathrm{mmol})$. After stirred for $20 \mathrm{~min}$, MTMS (2.24 mL, $16 \mathrm{mmol})$ was added and 0.32 $\mathrm{mL}$ of hydrochloric acid was added dropwise while stirring. Then the flask was sealed with thin films with pin holes and incubated for 2 days, so that gels could be formed. The product was filtrated and dried in a vacuum oven at $60{ }^{\circ} \mathrm{C}$ for $4 \mathrm{~h}$. The obtained material was crushed, grounded and sieved to obtain regularly sized particles between 30 and $60 \mu \mathrm{m}$ that were suitable for the evaluation and application of SPE. To ensure the complete removal of the templates, the materials were Soxhlet extracted with a mixture of ethanol/acetic acid $(8: 2, \mathrm{v} / \mathrm{v})$ for $24 \mathrm{~h}$ and then washed with copious ethanol/ acetic acid $(8: 2, \mathrm{v} / \mathrm{v})$. The MIP-HM was finally dried at $60^{\circ} \mathrm{C}$ in the vacuum for $24 \mathrm{~h}$. A similar procedure without template was used to prepare the non-imprinted polymer (NIP) as control material.
Static adsorption test: To measure adsorption capacity, $50 \mathrm{mg}$ of the MIP-HM or NIP was equilibrated with $10 \mathrm{~mL}$ of various concentrations of SAR dissolved in water. The mixture were mechanically shaken for $1 \mathrm{~h}$ at room temperature and separated by filtration. The filtrate was measured for the unextracted SAR by UV spectrometry. The equilibrium adsorption capacity (Q) was calculated according to eqn. 1: $\mathrm{Q}=\mathrm{V}\left(\mathrm{C}_{0}-\mathrm{C}_{\mathrm{e}}\right) / \mathrm{m}$, where $\mathrm{V}$ is the volume of solution $(\mathrm{mL}) ; \mathrm{C}_{0}$ and $\mathrm{C}_{\mathrm{e}}$ are the initial and the equilibrium concentration of SAR in water, respectively.

Molecularly imprinted solid phase extraction procedure: $50 \mathrm{mg}$ of the MIP-HM or NIP was packed into empty SPE cartridges, respectively and capped with fritted polyethylene disks at the top and bottom. After condition with the following solvents (in order): $3 \mathrm{~mL}$ of water/acetic acid 90:10 (v/v), $3 \mathrm{~mL}$ of water and $3 \mathrm{~mL}$ of methanol. $5.0 \mathrm{~mL}$ of SAR aqueous solution was passed through the cartridges at a flow rate of $0.5 \mathrm{~mL} \mathrm{~min}^{-1}$. Then the cartridge was washed with $5 \mathrm{~mL}$ of $\mathrm{H}_{2} \mathrm{O} /$ methanol $(90: 10$, v/v). The SAR was eluted with $2 \mathrm{~mL}$ of methanol-acetic acid-trifluoroacetic acid (90:9:1, v/v). The collected solution was dried using a gentle stream of nitrogen. The residues were redissolved in the mobile phase and analyzed by HPLC-UV. The non-molecularly imprinted polymers and $\mathrm{C}_{18}$ column were used as contrast experiment.

Determination of SAR in milk samples: Milk was selected for the spiked sample analysis. A $5 \mathrm{~mL}$ of milk containing LOM and SAR was placed in a $25 \mathrm{~mL}$ polypropylene tube and $0.25 \mathrm{~mL}$ of concentrated phosphoric acid and $10 \mathrm{~mL}$ of acetonitrile were added. The mixed sample was shaken for 6 $\mathrm{h}$ after the mixture was centrifuged for $10 \mathrm{~min}$ at $4000 \mathrm{rpm}$, the supernatant was filtered through a $0.22 \mu \mathrm{m}$ filter. The filtrate was concentrated at $45^{\circ} \mathrm{C}$ by rotary evaporators and stored in $4{ }^{\circ} \mathrm{C}$ for further work. The residues dissolved with water $(\mathrm{pH}$ 6.0 ) to $5 \mathrm{~mL}$ and filtration through a $0.22 \mu \mathrm{m}$ syringe filter, $5 \mathrm{~mL}$ of the filtrate was passed through the MIP-HCM cartridges. The above-mentioned MISPE procedure was used to separate and detect fluoroquinolones in milk. The milk samples were spiked with LOM and SAR at three concentration levels of $0.2,1.0,5.0 \mathrm{mg} \mathrm{kg}^{-1}$ and experiments were repeated three times.

\section{RESULTS AND DISCUSSION}

Characteristic of FT-IR spectra: To ascertain the presence of APTES and MTMS in the functionalized silica gel sorbents, FT-IR spectra were obtained from activated silica gel $\left(\mathrm{SiO}_{2}\right)$, non-imprinted polymer (NIP) and SAR-imprinted hybrid material (MIP-HM), respectively. As shown in Fig. 2, the observed features around 1635 and $3440 \mathrm{~cm}^{-1}$ indicated $\mathrm{O}-\mathrm{H}$ stretching vibrations. The bands around 775 and $469 \mathrm{~cm}^{-1}$ resulted from $\mathrm{Si}-\mathrm{O}$ vibrations. The $\mathrm{Si}-\mathrm{O}-\mathrm{Si}$ stretching vibration was reflected at $1026 \mathrm{~cm}^{-1}$. A characteristic feature of the SARimprinted and non-imprinted silica sorbents compared with activated silica-gel is is an aliphatic $\mathrm{C}$ - $\mathrm{H}$ bond and the stronger $\mathrm{CH}_{2}-\mathrm{N}$ bond, which therefore have a peak at $2972 \mathrm{~cm}^{-1}$ and a sharp peak at $1276 \mathrm{~cm}^{-1}$. It shows that there is not only the inorganic silica-gel, but also the saturated alkane and amino group in the materials. Compare with the NIP, the MIP-HM increases two peaks in 2360 and $1128 \mathrm{~cm}^{-1}$, which shows that there is template molecule in the imprinted material. 

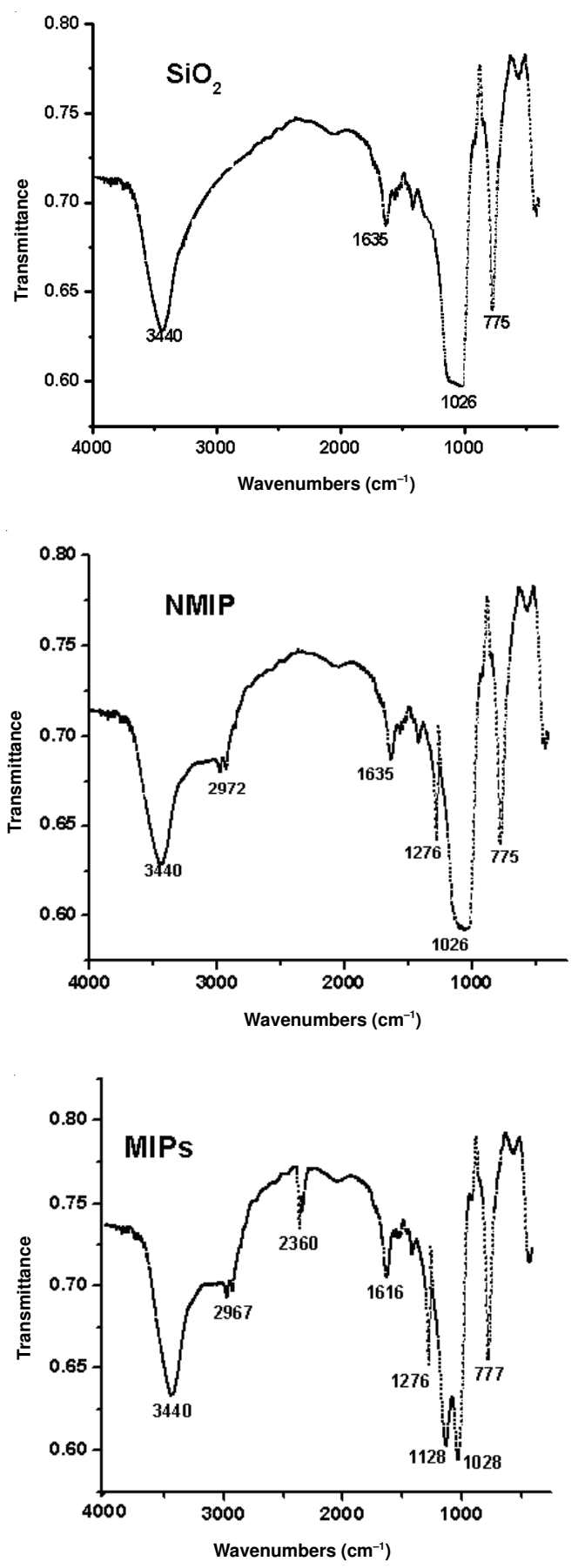

Fig. 2. FT-IR spectra of the $\mathrm{SiO}_{2}$, non-imprinted polymer (NIP) and SARimprinted polymer (MIPs)

Effect of organic silane on adsorption characteristics of the MIP-HM: The molar ratio of organic silane (APTES and MTMS) to TEOS is directly related to the moles of the active groups on the silica matrix. For the ratio of TEOS/ organic silane $<1$, the products are viscous colloids, or solid gels cannot be formed. The adsorption capacity of the sorbent prepared with ratios $>4$ is reduced. Proportion in about four, obvious mechanical strength and high adsorption capacity were observed. For the ratio of MTMS/APTES (Fig. 3), the adsorption capacity of the MIP-HM increased with increasing of MTMS, which may be attributed to the hydrophobic interaction increased gradually and the hydrophobic interaction electrostatic attractions are stronger between SAR and MIP-MS.

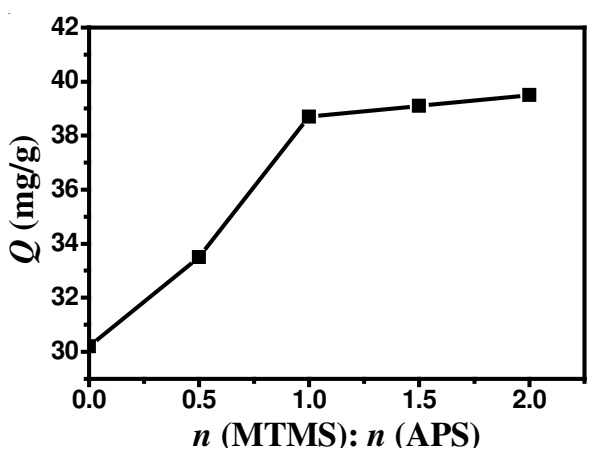

Fig. 3. Effect of the molar ratio of silane coupling agent on the binding capacity of SAR onto MIP-HM

However, when the proportion of MTMS/APTES > 1, the adsorption capacity increased trend abate, the hydrophobic interaction played a main role and its selectivity deceased. Therefore, the ratio of MTMS/APTES at 1 was chosen as the optimum condition.

Effect of $\mathbf{p H}$ on the MIP-HM: $50 \mathrm{mg}$ of the MIP-HM was equilibrated with $10 \mathrm{~mL}$ of $0.5 \mathrm{mg} / \mathrm{mL}$ aqueous solution of SAR. The solution $\mathrm{pH}$ was adjusted with $\mathrm{HCl}$ and $\mathrm{NaOH}$ solutions. The mixture were mechanically shaken for $1 \mathrm{~h}$ at room temperature and separated by centrifugation, the SAR in the supernatant was quantified by UV-VIS spectrophotometer. The optimization of $\mathrm{pH}$ value for adsorption medium plays a vital role in the adsorption studies. The effect of solution $\mathrm{pH}$ on the equilibrium adsorption capacities were showed in the Fig. 4. The SAR compound possesses amino group and carboxylic group, there are three protonation/deprotonation equilibrium. When $\mathrm{pH}$ is around 6.0, there is a protonated species of the amino group in SAR and the dissociated carboxylic groups exist in MIP-HM and SAR. The hydrophobic interaction and electrostatic attractions are stronger between MIP-HM and SAR. When $\mathrm{pH}<6.0$, as the $\mathrm{pH}$ value decreased, adsorption capacity decreased, which may be attributed to SAR has good hydrophilicity and the MIP-HM possesses hydrophobicity in the low $\mathrm{pH}$ value. When the $\mathrm{pH}$ was increased from $\mathrm{pH}$ 6-9, the adsorption capacity of the MIP-HM decreased. This may be due to the dissociated species of carboxylic groups exist in both MIP-HM and SAR, the electrostatic repulsions were not benefited for the adsorption system and the hydrophobic interaction became weak gradually between MIP-HM and SAR. Therefore, the equilibrium solution at $\mathrm{pH} 6$ was chosen as the optimum condition.

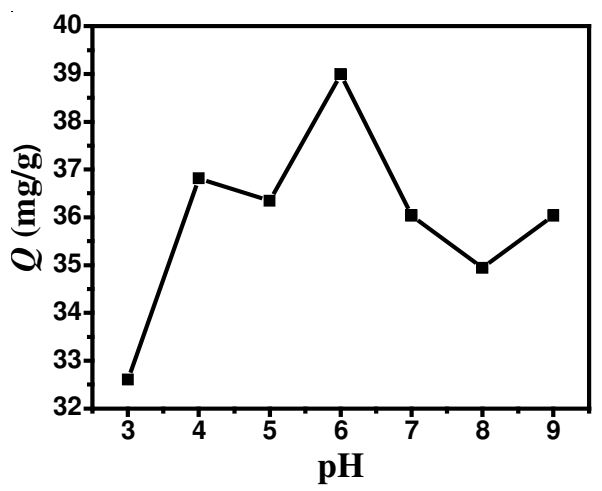

Fig. 4. Effect of different $\mathrm{pH}$ sample solutions on the binding capacity of SAR onto MIP-HM 
Binding study and Scatchard analysis: The static equilibrium adsorption experiments for the MIP-HM and NIP were carried out by varying the initial concentrations of SAR in the range of $0-3.00 \mathrm{mmol} \mathrm{mL^{-1 }}$. The binding isotherm of SAR onto MIP-HM was shown in Fig. 5a. The adsorption of MIP$\mathrm{HM}$ increased with increasing of the initial concentration. In general, the Scatchard plot is used for the evaluation of adsorption parameters. Furthermore, the Scatchard plot can indicate how many kinds of binding sites exist in the MIP-HM ${ }^{13}$. The average binding data of triplicate independent results can be linearly transformed according to the Scatchard equation $\left[\mathrm{Q} / \mathrm{C}_{\mathrm{e}}=\left(\mathrm{Q}_{\max }-\mathrm{Q}\right) / \mathrm{K}_{\mathrm{d}}\right]$. The results indicated that there were two different binding sites in the MIP-HM and only one kind of binding site in the NIP (Fig. 5b). In Fig. 5b, the fitting liner equation for the MIP-HM, the equilibrium dissociation constants $\left(\mathrm{K}_{\mathrm{d}}\right)$ of 72.5 and $290.2 \mathrm{mmol} \mathrm{L}^{-1}$ and the apparent maximum binding capacities $\left(\mathrm{Q}_{\max }\right)$ of 0.08665 and 0.1348 mmol g ${ }^{-1}$ could be calculated from the slope and the intercept of the linear equation.
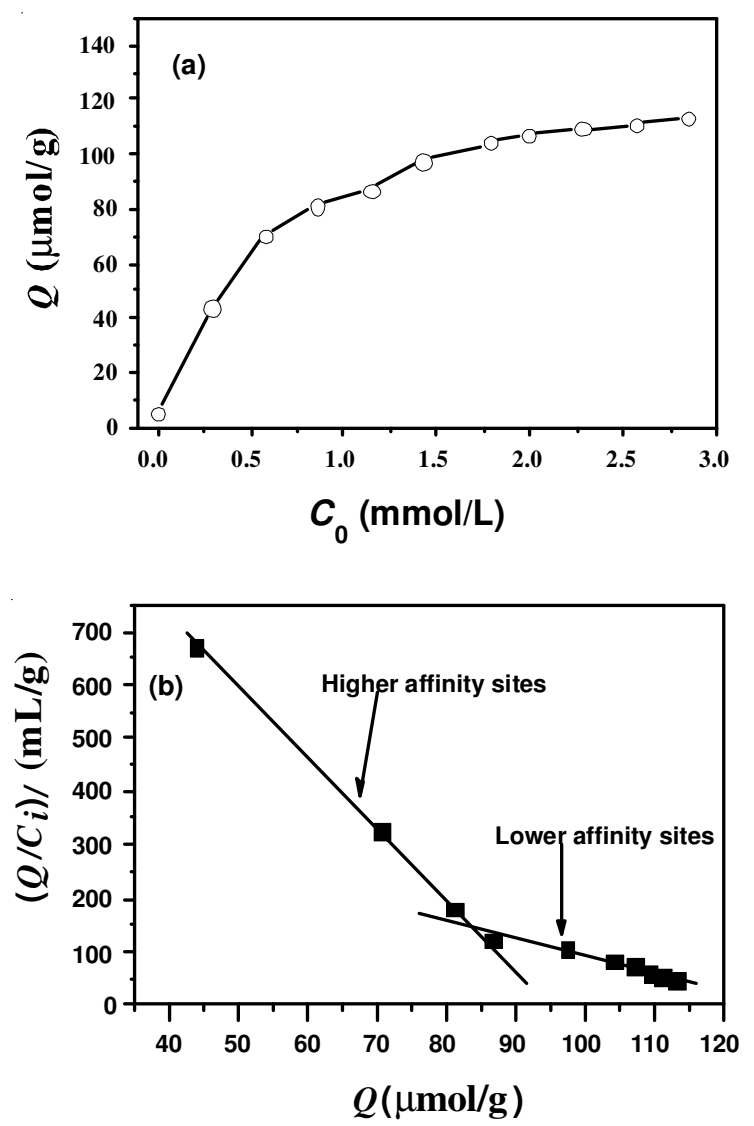

Fig. 5. Equilibrium binding curves (a) and the Scatchard analysis of the binding of SAR onto MIP-HM (b)

Molecular imprinted solid phase extraction: In order to optimize the selectivity of MISPE, conditioning, loading, washing and elution steps were evaluated and optimized. After pre-conditioning, the loading step was optimized. $2 \mathrm{~mL}$ of different loading solvents including methanol, water, dichloromethane and chloroform was passed through cartridges packed with $50 \mathrm{mg}$ of the MIP-HM. It was shown that SAR was retained on the MISPE column, water ( $\mathrm{pH}$ 6) was used as loading solution. In order to enhance the selectivity of
MIP-HM and decrease the cross-reactivity, the washing step was optimized. It is well known that the template could be retained on the MIP-HM by selective and nonspecific interactions. Thus, a washing solution with moderate elution strength was used to damage the nonspecific interactions and to let the target analyte be retained by specific interactions. After loading the cartridge with $2.0 \mathrm{~mL}$ of $1 \mathrm{mg} \mathrm{L}^{-1}$ of SAR aqueous solution, several solvents such as $\mathrm{H}_{2} \mathrm{O}, \mathrm{MeOH}$, DCM, chloroform and acetonitrile were investigated as washing solvents and the percentage of SAR removed from MIP-HM were $0,10,60$ and $80 \%$, respectively. Consequently, different proportions and volumes of toluene and methanol were investigated. The better result was obtained using $5 \mathrm{~mL}$ of $\mathrm{H}_{2} \mathrm{O} / \mathrm{MeOH}$ (90:10, v/v) as the washing solvents.

The eluting step was optimized based on the principle of elution that the analytes could be eluted completely by a small volume (1-2 mL) of strong solvent, while stronger than the analytes as much as possible to retain the impurities remain in the MISPE column. A series of elution solutions, water, $\mathrm{MeOH}$, ACN and TFA with HOAc in different proportions were used to optimize the eluting conditions. The best recovery was obtained by using $3 \mathrm{~mL}$ of MeOH-HOAc-TFA (90:9:1, v/v) as eluting solution. As shown in Fig. 6, they had the best eluting effect and the smallest effect.
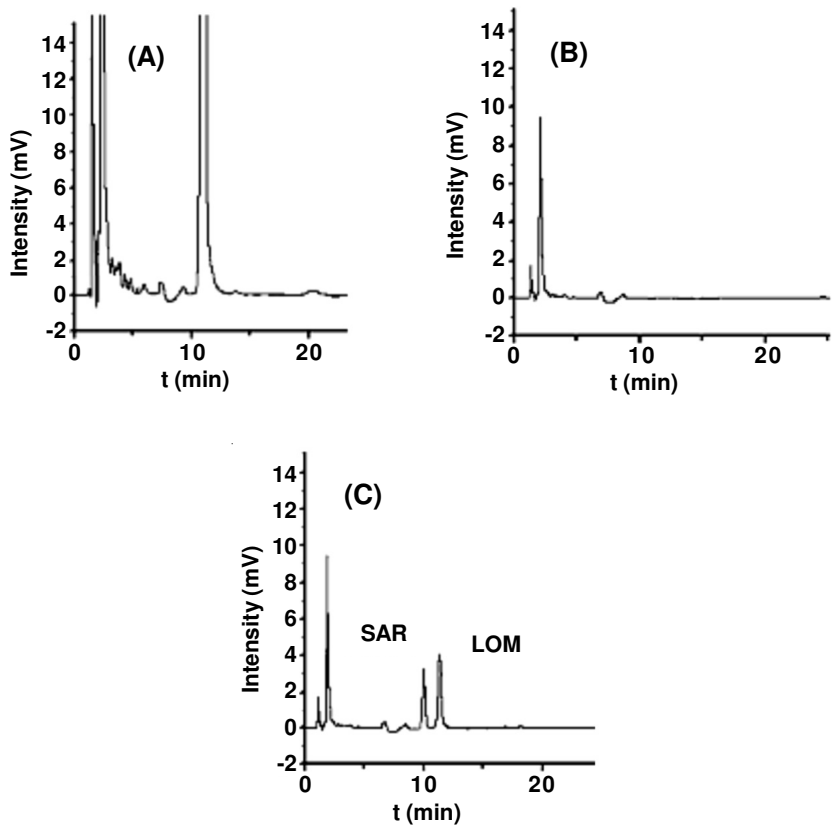

Fig. 6. Chromatograms obtained from the extraction of sarafloxacin and lomefloxacin from the milk samples: (A) the blank sample extract was percolated through the $\mathrm{C}_{18}$ cartridge; (B) the blank sample with a clean-up of MISPE and (C) the spiked milk with a clean-up of MISPE. Mobile phase: methanol-acetonitrile- $0.1 \%$ trifluoroacetic acid solution $\left(8: 20: 72\right.$, v/v). Flow rate: $1.0 \mathrm{~mL} \mathrm{~min}^{-1}$ at $25^{\circ} \mathrm{C}$. Samples spiked concentration: $1.0 \mathrm{mg} \mathrm{kg}^{-1}$. Injection volume: 10 $\mu \mathrm{L}$

Determination of fluoroquinolone residues in milk by MISPE-HPLC: In order to investigate the potential of the MIP-HM for the selective entrapment of target analyte from complex milk samples, satisfactory sample clean-up was achieved by the MISPE. Fig. 6 showed the chromatograms obtained for a blank milk sample extract was percolated 
TABLE-1

AVERAGE RECOVERIES (R), RELATIVE STANDARD DEVIATIONS (RSDS, N = 3), LIMIT OF DETECTION (LOD) AND LIMIT OF QUANTITATION (LOQ) OF SAR AND LOM OBTAINED AFTER MISPE OF THE SPIKED MILK SAMPLES

\begin{tabular}{ccccccc}
\hline Analyte & Spiked level $\left(\mathrm{mg} \mathrm{kg}^{-1}\right)$ & Detected $\left(\mathrm{m} \mathrm{kg}^{-1}\right)$ & $\mathrm{R}(\%)$ & $\mathrm{RSD}(\%)$ & $\mathrm{LOD}^{\mathrm{a}}\left(\mathrm{mg} \mathrm{kg}^{-1}\right)$ & $\mathrm{LOQ}^{\mathrm{b}}\left(\mathrm{mg} \mathrm{kg}^{-1}\right)$ \\
\hline \multirow{3}{*}{ SAR } & 0.2 & 0.104 & 75.60 & 4.23 & & \\
& 1.0 & 0.190 & 91.60 & 4.68 & 0.010 & 0.0333 \\
\hline \multirow{3}{*}{ LOM } & 5.0 & 0.491 & 94.20 & 3.56 & & 0.04 \\
& 0.2 & 0.082 & 61.73 & 5.21 & & 0.012 \\
\hline
\end{tabular}

${ }^{a} \mathrm{LOD}$ calculated as 3 times the signal-to-noise ratio; ${ }^{\mathrm{b}} \mathrm{LOQ}$ calculated as 10 times the signal-to-noise ratio.

through the $\mathrm{C}_{18}$ cartridge (Fig. 6a), a blank sample with a cleanup of MISPE (Fig. 6b) and a spiked milk with a clean-up of MISPE (Fig. 6c). As it can be observed in the chromatograms, after MISPE, the baselines' noises in Fig. $6 \mathrm{~b}$ were much less than those in Fig. 6a. It confirmed that satisfactory sample clean-up was achieved by the MISPE when applied to a complex matrix. $5 \mathrm{~g}$ of the milk samples was treated using the protocol described in the experimental section. The mean recoveries of SAR and LOM in milk evaluated by three spiking samples with different concentrations $\left(0.2,1.0\right.$ and $\left.5.0 \mathrm{mg} \mathrm{kg}^{-1}\right)$ were 75.6-94.2 and 61.73-88.56\%, respectively, with relative standard deviations (RSDs) of 3.56-5.21\% (Table-1). The limits of detection ( $\mathrm{LOD}, \mathrm{S} / \mathrm{N}=3$ ) and the limits of quantitation (LOQ, S/N = 10) of the proposed method were 0.010 and $0.0333 \mathrm{mg} \mathrm{kg}^{-1}$ for SAR, 0.012 and $0.04 \mathrm{mg} \mathrm{kg}^{-1}$ for LOM, respectively.

\section{Conclusion}

A new molecularly imprinted organic-inorganic hybrid polymer was prepared by sol-gel process in aqueous phase system based on the interaction of ion pair and hydrophobic between the sarafloxacin and the functional monomer. The binding capacity was evaluated by static adsorption and scatchard analysis, which showed that the synthesized molecularly imprinted sol-gel adsorbents not only have relatively good adsorption capacity and selectivity, but also have relatively purification complex matrix efficiency.

\section{ACKNOWLEDGEMENTS}

The authors gratefully appreciated the financial support by the Hebei Provincial Key Basic Research Program (No.
10967126D) and the Natural Science Foundation of Hebei Province (No. B2011201081).

\section{REFERENCES}

1. I. Choma, D. Grenda, I. Malinowska and Z. Suprynowicz, J. Chromatogr. $B$, 734, 7 (1999).

2. CFR-Code of Federal Regulations, Title 21, Part 556.: Tolerances For Residues of New Animal Drugs In Food. USA.

3 Council Regulation 2377/90/EEC Laying Down a Community Procedure for the Establishment of Maximum Residue Limits Veterinary Medicinal Products in Foodstuffs of Animal Origin, Off, J. Eur. Commun. L 224 (1990).

4 Bulletin of Ministry of Agriculture, P.R. China. No. 235. Veterinary Drug Maximum Residue Limits in the Food of Animal Origin, 12 (2002).

5 Q.F. Tang, T.T. Yang, X.M. Tan and J. Luo, J. Agric. Food Chem., 57, 4535 (2009).

6 H.-H. Chung, J.-B. Lee, Y.-H. Chung and K.-G. Lee, Food Chem., 113, 297 (2009).

7 A.V. Herrera-Herrera, J. Hernandez-Borges and M.A. RodriguezDelgado, J. Chromatogr. A, 1216, 7281 (2009).

8 G. Guo, W. Pan, D. Su and H. Chen, Chin. J. Chromatogr., 23, 401 (2005).

9 M.D. Marazuela and M.C. Moreno-Bondi, J. Chromatogr. A, 1034, 25 (2004).

10 P.Y. Liu, J. Shen, L. Gao, L. Liu, R. Li and Q. Li, Asian J. Chem., 22, 6275 (2010).

11 E. Rodriguez, M.C. Moreno-Bondi and M.D. Marazuela, J. Chromatogr. A, 1209, 136 (2008).

12 S. Bogialli, G. D'Ascenzo, A.D. Corcia, A. Laganà and S. Nicolardi, Food Chem., 108, 354 (2008).

13 Y.-K. Lv, L.-M. Wang, L. Yang, C.-X. Zhao and H.-W. Sun, J. Chromatogr. A, 1227, 48 (2012).

14 Y.-K. Lu and X.-P. Yan, Anal. Chem., 76, 453 (2004). 